\title{
Development of Magnetite Nano-composite Membrane for Membrane Defouling
}

\author{
N. A. Azmi', Q.H. Ng², A. L. Ahmad ${ }^{3} \&$ S. C. Low ${ }^{4}$ \\ ${ }^{1-4}$ School of Chemical Engineering Campus, Univcrsiti Sains Malaysia, Seri Ampangan, \\ 14300 Nibong Tebal S.1.S. Penang, Malaysia
}

\begin{abstract}
This research proposes to untangle the membrane fouling by introducing nanostructured magnetite $\left(\mathrm{Fe}_{3} \mathrm{O}_{4}\right)$ colloids into the polymeric membrane. In present study, nanostructure magnetite nanoparticles (MNPs) were synthesized via co-prccipitation method with ammonium hydroxide and sodium hydroxide as the precipitation agents at different $\mathrm{pH}$ condition. The synthesized MNPs were functionalized with poly(diallyldimcthylammonium chloride) (PDDA) and ther spin coated on the surface of the uitrafiltration cellulose acetate (CA) membrane. Intrinsic properties for this nano-composite membrane, in regards to the physical structures, surface negative charge density and the membranc filtration performance, on sufface fouling by humic acid solutions were investigated. Experimental results demonstrated that, the nano-composite membrane has significantly reduced the humic acid fouling on the membranc surface. This could be explainced by the electrostatic interactions between negatively charged humic acid moiecules and the nano-composite membrane. Throughout the study, the results provide some fundamental insights into the physical interactions that governing the membrane fouling during filtration.
\end{abstract}

Keywords: Fouling; magnetite nanoparticles, humic acids, nano-composite membrane, watcr treatment

\subsection{INTRODUCTION}

In recent years, various water treatment technologies are actively being pursued in both academic world and industry due to the rapid deterioration of water quality worldwide. The stringent regulations for drinking water quality have stimulated the membrane filtration to become one of the best alternatives that replacing the conventional drinking water treatment technologies [1-3].

Fouling remains a critical issue in many water filtration processes and serves as the dominant factor that restricts its widespread application $[3,4]$. The fouling causes a rapid irreversible loss of flux through the membrane which leads to the progressive deterioration of membrane performance [5].

\footnotetext{
* Corresponding to: S. C. Low (email: chsclow@eng.usm.ny)
}

Periodic hydraulic backwash procedures werc usually employed in reducing the effects of fouling. However, the used of hydraulic backwash is practical in removing the reversible foulant. Some deposited matter on the membrane surface and inside the pores cannot be removed and formed the irreversible part of fouling [6].

Recently, the synthesized of nanosized magnetic material have drawn much attention due to their unique properties and potential applications in biomedical $[7,8]$, heat transfer enhancement and environmental applications [9-11]. Magnetite $\left(\mathrm{Fe}_{3} \mathrm{O}_{4}\right)$ nanoparticles are preferred because of its high magnetic susceptibility, non-toxic, low cost instrument and low detection limit [12].

Currently the magnetic actuated membrane is an improvement of the membrane process in removing 
the foulant. The nano-composite membrane consist of a highly elastic polymer matrix with embedded nanosized of magnctic particles [13], which is sensitive to the magnetic field. In the presence of a local magnetic field, the magnetic membrane could be stiffened. This will reduced the object and membrane contact area and allowed the object to be either relocated or released.

The aimed of the present study is to untangle the fouling mechanism through magnetically actuated nanocomposite mcmbranc. The research efforts were dedicated to formularize a simple experimental protocol for surface functionalization of the polymeric membrane. Through electrostatic interaction between nanostructure magnetite $\left(\mathrm{Fe}_{3} \mathrm{O}_{4}\right.$ ) colloids and membrane, it provided the flexibility on the motion control of magnetite nanoparticles on the membrane surface. Defouling performance of the nano-composite membrane was tested accordingly by using humic acid substances as the foulant.

\subsection{RESEARCH METHODOLOGY}

\subsection{Materials}

A magnetite nanoparticle was supplied by NanoAmor (USA). Cellulose Acetate (CA) membrane used as the support membrane was supplied by GE Osmonic ((USA). Iron (III) chloride hexahydrate $\left(\mathrm{FeCl}_{3} \cdot 6 \mathrm{H}_{2} \mathrm{O}\right)$, iron (II) chloride tetrahydrate $\left(\mathrm{FeCl}_{2} \cdot 4 \mathrm{H}_{2} \mathrm{O}\right)$, sodium hydroxide $(\mathrm{NaOH})$ and hydrochloric acid fuming 37\% (HCL) were purchased from Merck (Darmstadt, Germany). Ammonium hydroxide solution 28\% in water $\left(\mathrm{NH}_{4} \mathrm{OH}\right)$ and poly(diallyl-dimethyl ammonium chloride) (PDDA) were supplied by Sigma-Aldrich (St. Louis, MO). All chemicals used in this study were analytical grade standards and used without further purification.

\subsection{Synthesis of $\mathrm{Fe}_{3} \mathrm{O}_{4}$ Nanoparticles}

The magnetite nanoparticles were prepared via $\mathrm{co}^{-}$ precipitation method. $4 \mathrm{~mL}$ of $0.5 \mathrm{M} \mathrm{FeCl}_{3} \cdot 6 \mathrm{H}_{2} \mathrm{O}$ was mixed with $1 \mathrm{~mL}$ of $1 \mathrm{M} \mathrm{FeCl}_{2} \cdot 4 \mathrm{H}_{2} \mathrm{O}$ at $25^{\circ} \mathrm{C}$ and stirred at stirring speed of $1100 \mathrm{rpm}$. A $25 \mathrm{~mL}$ of $\mathrm{NH}_{4} \mathrm{OH}$ or $\mathrm{NaOH}$ (with $\mathrm{pH}$ ranging from 9 to 14) that acted as the precipitating agent was immediately added into the mixture and stirred for $30 \mathrm{~min}$. Upon the completion of the co-precipitation process, the synthesized magnetite nanoparticles were collectcd by an $\mathrm{NdFeB}$ permanent magnet. Collected magnetite nanoparticles were washed with deionized water (DI) and centrifuged to ensurc that pure magnetite was obtained (repeated three times). The magnetites were then sonicated to allow monodisperse of magnctite in DI water.

\subsection{Functionalization of $\mathrm{Fe}_{3} \mathrm{O}_{4}$ Nanoparticles}

1000 ppm of magnetite nanoparticles suspension was prepared using DI water and sonicated. Similarly, $0.01667 \mathrm{~g} / \mathrm{mL}$ of PDDA was prepared and undergo sonication. The $\mathrm{pH}$ of both magnetitc nanoparticles (MNPs) suspension and PDDA solution was adjusted to $\mathrm{pH} 8.0$ to facilitate physisorption of PDDA on MNPs via electrostatic attraction for 1 day in rotator mixer.'The PDDA-coated magnetite nanoparticles were then collected using an $\mathrm{Nd}-\mathrm{FeB}$ permanent magnet and pre-washed before dispersed in DI water.

\subsection{Devclopment of Nano-composite Membrane}

The nano-composite membrane was prepared using Flat sheet CA membrane was placed onto the glass plate and fitted onto the vacuum chuck of spin coater. Subsequently, polymer solution containing PDDA-coated magnetite nanoparticles was dispersed onto the membrane surface at rotation speed of $300 \mathrm{rpm}$ and rotation time of $900 \mathrm{~s}$ until a homogeneous thin film polymer was formed. The nano-composite membrane was then air dried.

\subsection{Membrane Performance Test}

The performance of the nano-composite membrane toward reducing the nano-composite membranes were test. CA membranes were cut into the dimension of $1 \times 1 \mathrm{~cm}$ and labeled as sample 1 and sample 2 . Both samples were immersed in humic acid (HA) solutions for 13 hours, where the external magnetic field (magnetic bar) was imposed to the sample 1. The introduction of external magnetic field at sample 1 would induce magneto rotation motions of MNPs that bound on the membrane surface and further removed the deposited HA molecules. After 13 hours, the membrane samples were air dried for 24 hours prior analyzed using ATR-FTIR. 


\subsection{Characterization}

\subsubsection{Particle Size and Polydispersity Index of $\mathrm{Fe}_{3} \mathrm{O}_{4}$ Nanoparticles}

Average particle size and polydispersity index of magnetite nanoparticles were determined using DLS (Malvern Instruments Nanosizer ZS). The light scattering intensity autocorrelation function was fitted by the CONTIN algorithm to produce an intensityweighted distribution of hydrodynamic radii. For DLS measurement, the sample was prepared by dispersed the $0.01 \mathrm{ml}$ of magnetite suspension to $3.5 \mathrm{ml}$ of DI water.

\subsubsection{Attenuated Total Reflection Fourier Transform Infrared (ATR-FTIR)}

The fouling level of the nano-composite membrane was investigated using Thermo Scientific FTIR spectrometer system (NICOLET iS10, USA). The membranes spectra were analyzed using Diamond crystal over wavenumber range of $4000-600 \mathrm{~cm}^{-1}$ with 32 scans at $4 \mathrm{~cm}^{-1}$ resolutions.

\subsection{RESULTS AND DISCUSSION}

\subsection{Synthesis of Magnetite Nanoparticles}

Ammonium hydroxide and sodium hydroxide were selected as the precipitation agents to synthesis the magnetite nanoparticles under different $\mathrm{pH}$ condition (from $\mathrm{pH} 9$ to $\mathrm{pH} 14$ ). It was observed that, different precipitate colour were obtained when co-precipitation process were carried under the different $\mathrm{pH}$ conditions. Black precipitate solutions were observed at $\mathrm{pH} 11$ and pH 12 in Fig. 1 and pH 14 in Fig. 2. These solutions were shown to have magnetophoretic responses when imposed to the external magnetic field (magnetic bar). In this regards, the synthesized MNPs were magnetite responsive which is expected able to perform the magneto rotation motions when bound on the membrane surface and further detached the foulants away from the membrane surface.

However, clear yellow solutions were observed throughout the reaction of $\mathrm{NH}_{4} \mathrm{OH}$ at $\mathrm{pH} 9$ and $\mathrm{pH}$ 10 in Fig. 1 and $\mathrm{NaOH}$ from $\mathrm{pH} 9$ and $\mathrm{pH} 12$ in Fig. 2. These yellow colour solutions were the intermediate form of solutions due to the insufficient of precipitation agent. This intermediate forms would change if the precipitation agent continuously added to the process

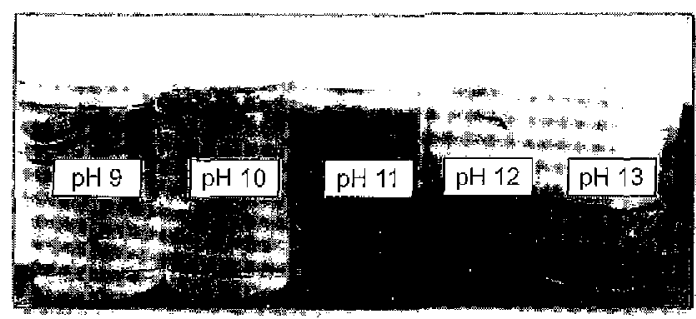

Figure 1 Magnetite nanoparticle prepared at different $\mathrm{pH}$ of ammonium hydroxide $\left(\mathrm{NH}_{4} \mathrm{OH}\right)$

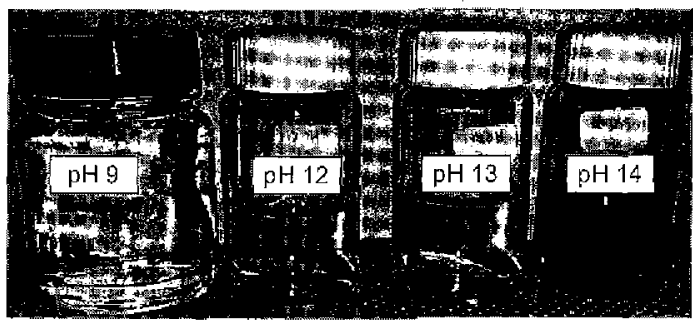

Figure 2 Magnetite nanoparticle prepared at different $\mathrm{pH}$ of sodium hydroxide $(\mathrm{NaOH})$

until the $\mathrm{OH}$ - ions sufficient to form MNPs. Knowing that, $\mathrm{NaOH}$ solution contained of higher density of $\mathrm{OH}^{-}$than NI- $\mathrm{I}_{4} \mathrm{OH}$ solution with the same molarities. However the used of strong alkaline media such as $\mathrm{NaOH}$, can caused the formation of non-magnetic iron compound [14] such as $\alpha-\mathrm{FeOOH}$ and other ion compounds [1.5].

Fluctuate trends were observed for both hydrodynamic diameter (Fig. 3) and PDI (Fig. 4) of MNPs when $\mathrm{NH}_{4} \mathrm{OH}$ was used as the precipitation agent in co-precipitation process. The interesting finding is that both highest and lowest readings were found at the narrow pH range within $\mathrm{pH} 11$ to $\mathrm{pH} 12$, respectively. MNPs showed to have the average hydrodynamic size of $1046.4 \pm 200 \mathrm{~nm}$ at $\mathrm{pH} 11$ and only $132.1 \pm$ $0.55 \mathrm{~nm}$ at $\mathrm{pH}$ 12. PDI was also followed the trend of bydrodynamic size where showed to be 0.798 at $\mathrm{pH} 11$ and monodisperse 0.253 at $\mathrm{pH} 12$. The monodisperse of MNPs at $\mathrm{pH} 12$ might due to electrostatic interaction that leads the repulsion between the negatively charge MNPs. MNPs carry negative surface charge at $\mathrm{pH}$ higher than its point zero charge $[16,17]$.

As for $\mathrm{NaOH}, \mathrm{MNPs}$ were only able to produce at $\mathrm{pH} 13$ or higher. This might be due to the density of $\mathrm{OH}^{-}$ions in the $\mathrm{NaOH}$ solution at certain $\mathrm{pH}$. It is well known that $\mathrm{pH}$ value is responsible for the charge density of $\mathrm{OH}$ ions, which responsible for the formation of $\mathrm{MNP}_{\mathrm{s}} \mathrm{NaOH}$ solution at $\mathrm{pH}$ below 12 resulted to have low density of ions $\mathrm{OH}^{-}$, thus, restricted 


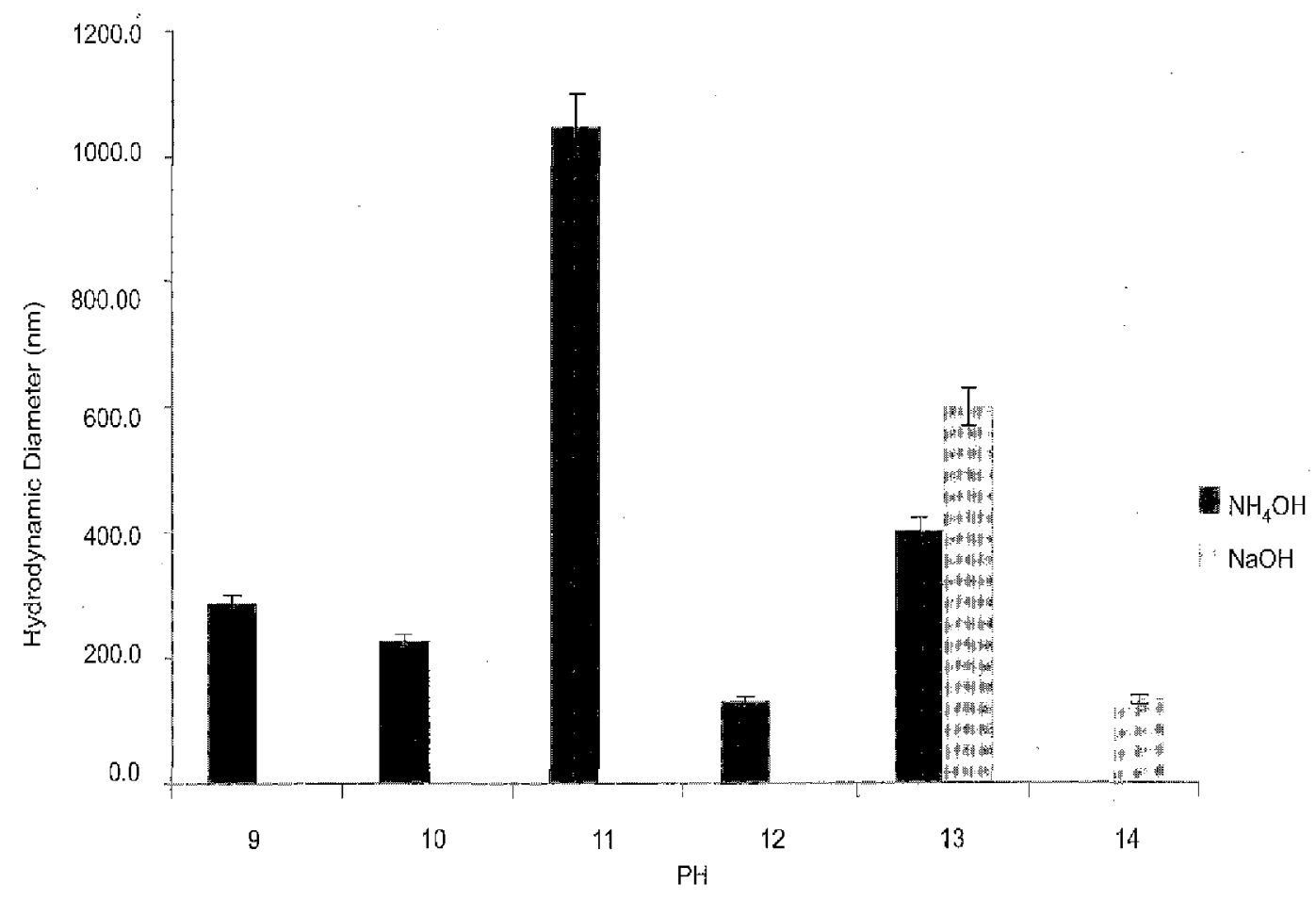

Figure 3 Hydrodynamic diameter of magnetite nanoparticle synthcsize at different $\mathrm{pH}$ ratio and precipitation agent

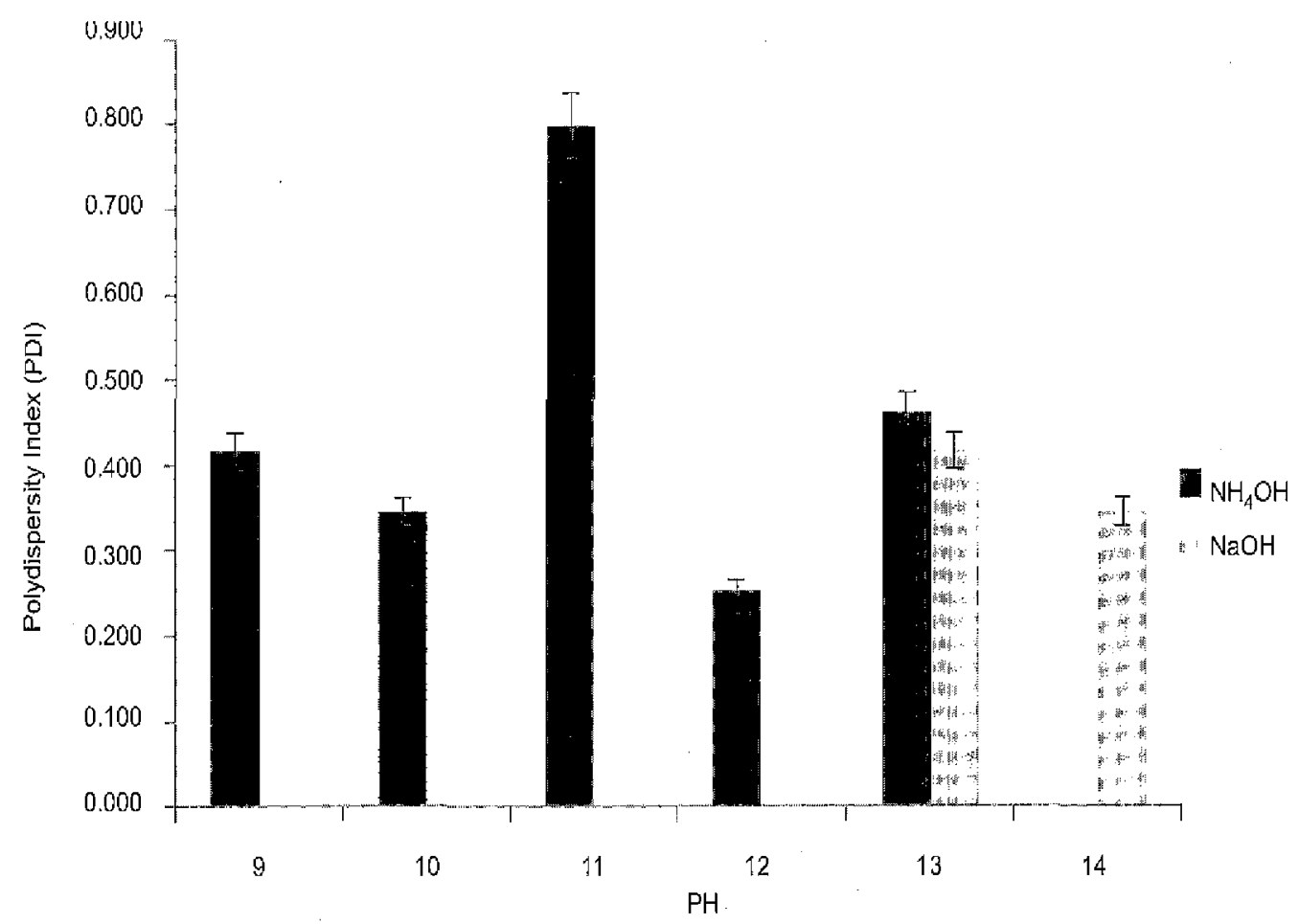

Figure 4 PDI of magnetite nanoparticle synthesize at different $\mathrm{pH}$ ratio and precipitation agent 
the formation of magnetic responsive nanoparticles and maintained as intermediate form of solutions. However, the intercst was focused on $\mathrm{pH} 14$ since the MNPs were magnetite responsive (black color of solution) under this $\mathrm{pH}$ condition. At $\mathrm{pH} 14$, the produced MNPs appeared in small hydrodynamic diameters of $134.2 \pm$ $2.27 \mathrm{~nm}$ and low PDI of 0.345 . Under the consideration of both small hydrodynamic diameter and low PDI, both $\mathrm{NH}_{4} \mathrm{OH}$ and $\mathrm{NaOH}$ were giving the similar characteristics of produced MNPs. However, $\mathrm{NH}_{4} \mathrm{OH}$ was selected as precipitation agent for further study due to the lower $\mathrm{pH}$ condition ( $\mathrm{pH}$ 12) was preferred for membrane filtration process.

\subsection{Defouling Performance of Magnetite Nano- composite Membrane}

Membrane defouling study was carried out by immersing the membrane sample in HA solution for 13 hours. The fouling level of HA onto membrane surface was analyzed using ATR-FTIR spectrophotocopy, as shown in Fig. 5. Samples 1 (membrane that exposed to the external magnetic fieid) and sample 2 (membrane that did not exposed to the external magnetic field) showed two significantly different transmission peaks at 1733 and $1636-1581 \mathrm{~cm}^{-1}$ wavenumber respectively.

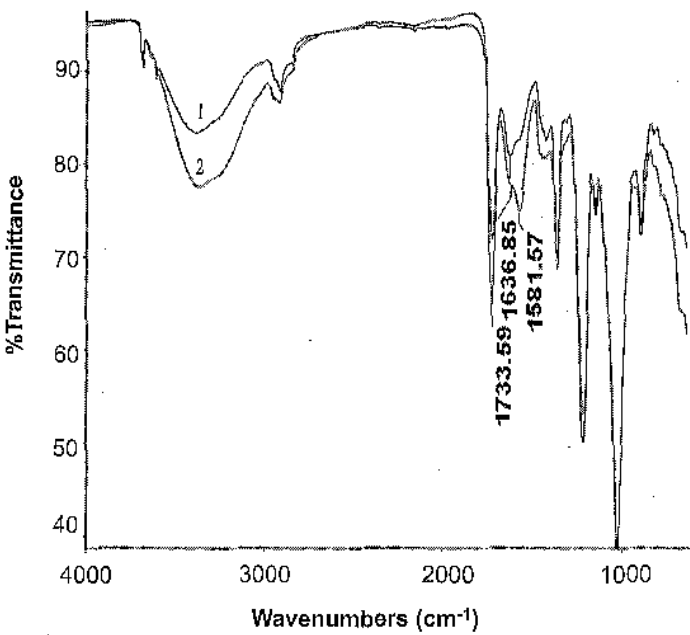

Figure 5 ATR-FTIR spectra for nano-composite membrane (1) with and (2) without the introduction of external magnetic field

The peak at $1733 \mathrm{~cm}^{-1}$ could be attribute to the presence of $\mathrm{C}=\mathrm{O}$ stretching in carboxylic acid groups.
The peak at 1636 and $1581 \mathrm{~cm}^{-1}$ attribute to the presence of $\mathrm{C}=\mathrm{C}$ and $\mathrm{N}-\mathrm{H}$ group respectively. At peak of $1733 \mathrm{~cm}^{-1}$, sample 1 with the exposed to the external magnetic field was found to have a stronger absorption band and vice versa at the overlapping peak of $1581-1636 \mathrm{~cm}^{-1}$. Based on these two peaks, it shows that the membrane sample 2 contained highes number of HA molecules that deposited on the membrane surface. These prove that, magnetite nano-composite membrane with the introducing of external magnetic field was could detached the HA precipitation on the membrane surface, thus, reduced the membrane fouling. When the magnetic nano-composite membrane was exposed to the external magnetic field, it will promote the alignment of MNPs that bound onto the membrane surface in one direction. In this regards, the magneto-induced rotation motions within the membrane polymer matrix were performed if two opposite directions were take placed in sequence. The rotational motions of the MNPs, promoted the detachment of the HA from the CA membrane. In turn, HA could easily remove from the MNPs and membrane surface and reduce fouling.

\subsection{CONCLUSION}

In present study, MNPs with small hydrodynamic diameter and polydispersity index were successfully synthesis by using $25 \mathrm{~mL}$ of $\mathrm{NH}_{4} \mathrm{OH}$ at $\mathrm{pH} 12$ with average hydrodynamic diameters of $132.1 \mathrm{~nm}$ and PDI of 0.253 . The synthsised MNPs were further stabilized using PDDA and coated onto CA membrane to form magnetic nano-composite membrane. The results demonstrated high efficiency of the removal of humic acid from the membrane surface, in the advantage that the movement of the magnetically responses of MNPS on the membrane surface suppresses fouling.

\section{ACKNOWLEDGMENT}

The authors wish to thank the financial support granted by The Institution of Higher Education ERGS grant (6730013) and USM Membrane Science and Technology Cluster. 


\section{REFERENCES}

[1] Z. Domany, et al. 2002. Humic substances removal from drinking water by membrane filtration. Desalination. $145: 333-337$.

[2] V. Siyanytsya, et al. 2008. Natural organic matter removal from water by complexationultrafiltration. Desalination. 223 : 91-96.

[3] X. Fu, et al. 2008. Effect of surface morphology on membrane fouling by humic acid with the use of cellulose acetate butyrate hollow fiber membranes. Journal of Membrane Science. 320 : 483-491.

[4] D. B. Mosqueda-Jimenez, et al. 2008. Fouling characteristics of an ultrafiltration membrane used in drinking water treatment. Desalination. $230: 79-91$.

[5] K. Katsoufidou, et al. 2005. A study of ultrafiltration membrane fouling by humic acids and flux recovery by backwashing: Experiments and modeling. Journal of Membrane Science. $266: 40-50$.

[6] G.F.Crozes, et al. 1997. Impact of ultrafiltration operating conditions on membrane irreversible fouling. Journal of Membrane Science. 124 : 63-76.

[7] J. Mürbe, et al. 2008. Synthesis and physical characterization of magnetite nanoparticles for biomedical applications. Materials Chemistry and Pbysics. $110: 426-433$.

[8] K. Nishio, et al. 2007. Preparation of sizecontrolled magnetite nanoparticles for biomedical applications. Journal of Magnetism and Magnetic Materials. $310: 2408-2410$.

[9] X. M. Li, et al. 2011, Magnetic Fe 304 nanoparticles: Synthesis and application in water treatment. Nanoscience and Nanotechnology - Asia. $1: 14-24$.
[10] W. Yantasee, et al. 2007. Removal of heavy metals from aqueous systems with thiol functionalized superparamagnetic nanoparticles. Environmental Science and Technology. 41 : 5114-5119.

[11] F. Ge, et al. 2012. Effective removal of heavy metal ions $\mathrm{Cd} \mathrm{2+,} \mathrm{Zn} \mathrm{2+,} \mathrm{Pb} 2+, \mathrm{Cu} 2+$ from aqueous solution by polymer-modified magnetic nanoparticles. Journal of Hazardous Materials. 211-212:366-372.

[12] H. Guo, et al. 2010. Application of magnetic nanoparticles for UF membrane integrity monitoring at low-pressure opcration. Journal of Membrane Science. 350 : 172-179.

[13] C. Schlemmer, et al. 2009. The design of thin polymer membranes filled with magnetic particles on a microstructured silicon surface. Nanotechnology. 9.

[14] G. Gnanaprakash, et al. 2007. Effect of initial $\mathrm{pJ}$ and temperature of iron salt solutions on formation of magnetite nanoparticles. Materials Chemistry and Pbysics. 103 : 168-175.

[15] I. Martínez-Mera, et al. 2007 Synthesis of magnetite ( $\mathrm{Fe} 3 \mathrm{O} 4)$ nanoparticles without surfactants at room temperature. Materials Letters. $61: 4447-4451$.

[16] J.-D. Hu, et al. 2010. Effect of dissolved organic matter on the stability of magnetite nanoparticles under different $\mathrm{pH}$ and ionic strength conditions. Science of The Total Environment. $408: 3477-3489$.

[17] E. Illés and E. Tombácz. 2006. The effect of humic acid adsorption on $\mathrm{pH}$-dependent surface charging and aggregation of magnetite nanoparticles. Journal of Colloid and Interface Science, $295: 115-123$. 